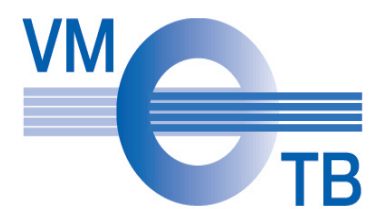

Die Ziele der VMTB

$\nabla$

Liebe KollegInnen, in Deutschland wurde 2001 die Vereinigung medizinisch-technischer Berufe in der DRG (VMTB) gegründet. Einige der Vorstandsmitglieder gehören auch dem Herausgeberteam dieser neuen Fachzeitschrift für RadiologietechnologInnen und MTRAs an. Da wir von vielen Kollegen noch immer als zweiter Berufsverband wahrgenommen werden, möchte ich an dieser Stelle die Möglichkeit nutzen in der ersten Ausgabe der Radiopraxis einmal die Ziele dieser Fachvereinigung für medizinisch-technische RadiologieassistentInnnen vorzustellen.

\title{
Kein Berufsverband
}

Wir sind zwar ebenso wie der deutsche Verband technischer Assistenten in der Medizin (DVTA) mit Angeboten zur Fort- und Weiterbildung der Kollegen betraut, allerdings ausschließlich auf dem Gebiet der diagnostischen Radiologie - aber wir sind kein Berufsverband. Der DVTA wird von uns als alleiniger Berufsverband der MTA aller Fachrichtungen anerkannt und in all seinen berufspolitischen Aktivitäten unterstützt.

Enge Zusammenarbeit mit den Radiologen Die VMTB ist eine Gruppierung von MTRAs innerhalb der deutschen Röntgengesellschaft (DRG). Wir werden von den Radiologen unterstützt und möchten die gute Zusammenarbeit und den partnerschaftlichen Umgang miteinander ausbauen und das in uns gesetzte Vertrauen rechtfertigen. Die Radiologen und die MTRAs haben viele gemeinsame Ziele. Durch die neuen Rahmenbedingungen im Gesundheitswesen werden an die Arbeitgeber zunehmend Qualitätsanforderungen gestellt, die einerseits ihre eigene Qualifikation betreffen, aber andererseits auch die ihrer Mitarbeiter. Somit ist ein gemeinsames Ziel sicherlich die ständige Fort- und Weiterbildung aller in der Radiologie tätigen Fachkräfte.

Geschäftsstelle: VMTB - Vereinigung der Medizinisch-Technischen Berufe in der DRG Frau Sonja Müller Straße des 17. Juni 114 10623 Berlin Tel.: +49 (0) 3091607015 Fax: +49 (0) 3091607022 E-mail:mueller@drg.de www.drg.de/data/VMTB/ home.html

\section{Fortbildung als wichtigstes Ziel}

Das vorrangige Ziel der VMTB ist es unseren in der Diagnostik tätigen KollegInnen praxisbezogen und effektiv Fortbildungen anzubieten, die regional veranstaltet werden. Unsere Fortbildungen sind von MTRAs und Radiologen für unsere Berufsgruppe konzipiert. Sie sind überwiegend als Hand's-on-Kurse für eine begrenzte Teilnehmerzahl ausgewiesen damit sie auf die Bedürfnisse der teilnehmenden KollegInnen eingehen können.
Wir sind dabei, die Kurse ständig auszubauen, um sie Ihnen an regional unterschiedlichen Standorten anbieten zu können, dazu haben wir bereits die ersten Kontakte geknüpft.

Liebe KollegInnen, ich hoffe Ihnen durch die Vorstellung unserer Vereinigung anlässlich der Neuerscheinung der Fachzeitschrift Radiopraxis ein paar wissenswerte Informationen gegeben zu haben.

Ursula Saß

Rückblick auf den 37. Fortbildungskurs („Lebkuchenkongress“) für ärztliches Assistenzpersonal im Dezember 2007 in Nürnberg

$\checkmark$

Vieles hat sich im Laufe der letzten Jahre geändert. Seit dem Jahr 2002 ist es möglich, die Fachkunde/Kenntnisse im Strahlenschutz zu aktualisieren. 2005 kam der große Umzug von der Erziehungswissenschaftlichen Fakultät in das CongressCenter Nürnberg Ost. Im letzten Jahr wurde unser „Lebkuchenkongress“ das erste Mal in Zusammenarbeit zwischen den radiologischen Instituten des Klinikums Nürnberg und der Universität Erlangen-Nürnberg, der Strahlenschutzkursstätte Nürnberg-Erlangen und der Vereinigung der Medizinisch-Technischen Berufe in der DRG (VMTB) durchgeführt. Dank der Unterstützung vieler fleißiger Helfer gelang es uns auch diese Mal wieder attraktive Themen für Kollegen aus der Röntgendiagnostik, Strahlentherapie und der Nuklearmedizin anzubieten. Das wissenschaftliche Programm wurde von über 300 Teilnehmern besucht und in den Klinikseminaren konnten mehr als 100 Teilnehmer "hands on“ ihr Wissen erweitern. Auf Grund der Umstrukturierung in der Organisation des Kongresses traten insbesondere bei der Registrierung der Teilnehmer Probleme auf. Dies bedauern wir sehr. An dieser Stelle möchten wir uns für Ihre konstruktive Kritik bedanken. Nur so ist es möglich, die Veranstaltung für Sie zu perfektionieren und deren Attraktivität zu steigern. In diesem Zusammenhang möchten wir darauf hinweisen, dass für den diesjährigen „Lebkuchenkongress“, welcher vom 5.-6. Dezember 2008 stattfindet, die Anmeldung ausschließlich über die Homepage der DRG erfolgen wird. Wir würden uns freuen, sie wieder zahlreich begrüßen zu dürfen.

Katja Röhr 